REGARDS

SUR L'ECONOMIE ALLEMAND

BULLETIN ECONOMIQUE DU CRAC
Regards sur l'économie allemande

Bulletin économique du CIRAC

118-119| 2015

Varia

\title{
Priorité : investir « dans les têtes »
}

Isabelle Bourgeois

\section{OpenEdition}

Journals

Édition électronique

URL : http://journals.openedition.org/rea/4881

DOI : 10.4000/rea.4881

ISSN : 1965-0787

Éditeur

CIRAC

\section{Édition imprimée}

Date de publication : 31 décembre 2015

Pagination : $3-4$

ISSN : 1156-8992

\section{Référence électronique}

Isabelle Bourgeois, «Priorité : investir « dans les têtes » », Regards sur l'économie allemande [En ligne],

118-119 | décembre 2015, mis en ligne le 31 décembre 2017, consulté le 15 septembre 2020. URL

http://journals.openedition.org/rea/4881 


\section{Priorité : investir " dans les têtes "}

L'économie allemande poursuit sur son rythme de croisière. Bien qu'au $3^{e}$ trimestre, le PIB n'ait progressé que de 0,3\% (Destatis), sur l'ensemble de l'année en cours, il devrait, de l'avis unanime des experts, croître de 1,7\%. En 2016, la croissance devrait se situer dans une fourchette comprise entre 1,6 \% (Conseil des Sages) et $2 \%$ (KfW).

Dans son rapport d'automne présenté le 8 octobre, le Groupe de travail des instituts économiques, qui prévoit un taux de 1,8\% sur ces deux années, souligne que si la dynamique de l'économie allemande a définitivement surmonté le creux laissé par la récession de 2009 , et si ses fondamentaux pourraient laisser présager une croissance plus forte, cette dernière se trouve bridée par un ensemble de facteurs. L'économie mondiale, principalement celle des pays émergents, s'est ralentie ; celle de la zone Euro, toujours aux prises de la crise de la dette souveraine, continue de stagner. Quant à la dépréciation de l'Euro face au Dollar, ses effets dynamisants sur la compétitivité-prix et donc la balance commerciale n'ont été qu'éphémères.

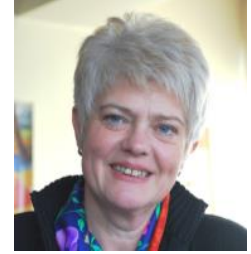

Isabelle Bourgeois, Rédactrice en chef

\begin{tabular}{|c|c|c|c|c|}
\hline \multicolumn{5}{|c|}{ Prévisions du Conseil des Sages } \\
\hline & 2013 & 2014 & $2015 P$ & $2016 P$ \\
\hline \multicolumn{5}{|l|}{ évolution } \\
\hline PIB (\%) & 0,3 & 1,6 & 1,7 & 1,6 \\
\hline Consommation (\%) & 0,7 & 1,1 & 1,9 & 1,8 \\
\hline privée (\%) & 0,6 & 0,9 & 1,9 & 1,8 \\
\hline publique (\%) & 0,8 & 1,7 & 2,1 & 2,0 \\
\hline \multicolumn{5}{|l|}{ Investissements } \\
\hline biens d'équipement (\%) & $-1,3$ & 3,5 & 2,4 & 2,7 \\
\hline construction (\%) & $-1,1$ & 2,9 & 1,0 & 2,0 \\
\hline autres (\%) & $-0,3$ & 3,1 & 2,7 & 3,0 \\
\hline Demande intérieure (\%) & 0,8 & 1,3 & 1,4 & 1,9 \\
\hline Part de l'excédent commercial dans le PIB (points de pourcentage) & $-0,5$ & 0,4 & 0,4 & $-0,1$ \\
\hline exportations (\%) & 1,6 & 4,0 & 6,2 & 4,5 \\
\hline importations (\%) & 3,1 & 3,7 & 6,3 & 5,7 \\
\hline Productivité horaire (\%) & n.c. & 0,4 & 0,9 & 0,6 \\
\hline Prix à la consommation (\%) & 1,5 & 0,9 & 0,3 & 1,2 \\
\hline Revenu disponible des ménages (\%) & n.c. & 2,3 & 2,7 & 2,9 \\
\hline \multicolumn{5}{|l|}{ état } \\
\hline Actifs occupés (milliers) & 42328 & 42703 & 43021 & 43333 \\
\hline Actifs occupant un emploi soumis à cotisations sociales (milliers) & 29713 & 30197 & 30803 & 31257 \\
\hline Chômeurs inscrits (milliers)*) & 2950 & 2898 & 2801 & 2891 \\
\hline Taux de chômage (Agence fédérale pour l'emploi) (\%) & 6,9 & 6,7 & 6,4 & 6,6 \\
\hline Taux de chômage (Destatis, critères OIT) (\%) & 5,2 & 5,0 & 4,6 & 4,6 \\
\hline Déficit public (\% du PIB) & $-0,1$ & 0,3 & 0,7 & 0,2 \\
\hline Dette publique (\% du PIB) **) & n.c. & 74,9 & 70,8 & 67,8 \\
\hline
\end{tabular}

Source : Sachverständigenrat zur Begutachtung der gesamtwirtschaftlichen Entwicklung, Jahresgutachten 2015/16 (11-11-2015). NB : PIB et composantes en données corrigées des prix. *) Données administratives de l'Agence fédérale pour l'emploi (Nuremberg); ${ }^{*}$ ) critères du Traité de Maastricht.

Malgré un excédent de près de 209 milliards $€$ sur les dix premiers mois de l'année, la balance commerciale contribue peu à la croissance actuellement. Au $3^{\mathrm{e}}$ trimestre, elle a même tassé celle-ci de 0,4 point, les importations ayant augmenté nettement plus $(+1,1 \%)$ que les exportations $(+0,2 \%)$ en comparaison avec le trimestre précédent (Destatis). Après le creux du premier semestre, les carnets de commande de l'industrie tendent à nouveau à se remplir ( $+1,8 \%$ en octobre ; Destatis) ; les commandes proviennent tant des marchés extérieurs, notamment européen, que domestique. La demande porte comme toujours sur les biens d'investissement $(+2,7 \%)$, mais aussi sur les biens de consommation $(+3,8 \%)$. La relative morosité de la conjoncture mondiale et européenne n'en incite pas moins les industriels à la prudence en matière d'investissement, d'autant que le taux d'utilisation des capacités est supérieur à la moyenne, comme le souligne la Bundesbank (rapport mensuel de novembre).

La conjoncture allemande est tirée par la consommation intérieure, et notamment celle des ménages. En effet, le marché du travail est toujours aussi dynamique, ce qui entretient la confiance en l'avenir. Le taux de chômage est historiquement bas en ... la consommation est le seul moteur conjoncturel

Malgré un fort excédent commercial, ... comparaison internationale $(4,6 \%)$, et la main-d'œuvre qualifiée - principalement in- 
La rigueur budgétaire permet de faire face à l'imprévu
Investir dans les " têtes bien faites" génieurs et techniciens - est très recherchée par les entreprises. Les faibles prix pétroliers réduisent la facture énergétique des Allemands comme leurs frais de carburant, ce qui dope leur pouvoir d'achat, de même que la hausse des transferts sociaux et l'augmentation des salaires nets enregistrées en 2015 (2,8\%; Destatis). Cette dernière découle en partie seulement des négociations collectives; l'introduction du salaire minimum légal au $1^{\text {er }}$ janvier 2015 a en effet eu un impact non négligeable sur les salaires des actifs faiblement qualifiés ou occupés dans des secteurs à faible qualification au cours du premier semestre, surtout dans l'est de l'Allemagne : ils ont progressé trois fois plus vite que ceux des salariés qualifiés et des cadres, estiment la Bundesbank et le Conseil des Sages.

Même si certains facteurs conjoncturels ont contribué à doper l'économie allemande, celle-ci reste toujours structurellement bien positionnée. Certes, la politique de redistribution du gouvernement fédéral était limitée dans le temps, certes, la faiblesse des taux d'intérêts qui avait soutenu l'investissement prendra fin un jour, l'Euro finira par sortir de sa faiblesse face au Dollar, et la facture énergétique va se renchérir. Mais bien que, au-delà des risques géopolitiques, le contexte réservé à l'activité se présente moins porteur à partir de 2016, la croissance ne devrait pas trop en pâtir. Car les fondamentaux de l'économie allemande sont solides, grâce notamment aux réformes de la protection sociale et du marché du travail des précédents gouvernements. Quant à la politique de consolidation budgétaire menée avec constance, elle permet de faire face à l'imprévu comme l'afflux d'un million de réfugiés en l'espace d'un an seulement. La Bundesbank ne manque pas de le relever dans son rapport de novembre : ce défi exceptionnel "révèle l'avantage que présentent un budget structurel assez favorable, ainsi que l'existence de marges de sécurité par rapport aux plafonds fixés par les règles budgétaires européennes et nationales». Elles permettent en effet à l'Allemagne de réaliser dans l'immédiat les dépenses liées à l'afflux des réfugiés tout en respectant à la fois le Pacte de stabilité et de croissance et la « règle d'or » nationale.

MAIS CELA NE SUFFIT PAS. IL S'AGIT DÉSORMAIS DE FAIRE EN SORTE QUE le modèle économique et social allemand soit à même de relever les défis du futur. Ce point est au centre aussi bien du rapport d'automne des instituts économiques que du rapport annuel du Conseil des Sages, présenté un mois plus tard. Ce dernier, qui a intitulé son rapport «Enjeu prioritaire: préparer l'avenir » (Zukunfstfähigkeit in den Mittelpunkt), rappelle l'urgence de la poursuite de la réforme du système de retraites ou d'une réforme de fond de la fiscalité, et insiste tout particulièrement sur deux priorités : l'achèvement de la construction de la zone Euro (il y avait consacré un rapport thématique cet été) et l'intégration des réfugiés dans l'économie et la société allemandes. En effet, "l'intégration dans le marché du travail est un aspect important de l'intégration dans la société » et une garantie de cohésion sociale. Mais la nouvelle main-d'œuvre doit impérativement être qualifiée pour ne pas peser sur une productivité affaiblie par l'insertion massive en emploi d'une main-d'œuvre peu qualifiée depuis les grandes réformes du marché du travail qui ont accru le taux d'activité. II ne s'agit pas de lancer des "programmes publics d'investissement ", mais au contraire "d'améliorer les conditions-cadre » réservées à l'activité, seules à même d'accroître la productivité

Ce plaidoyer pour plus de réformes structurelles n'exclut nullement une hausse des investissements publics - en priorité dans le système d'éducation et de formation, une approche partagée par les instituts économiques qui développent tout particulièrement ce point. Et qui soulignent que ce qui importe, "ce n'est pas le montant des moyens engagés, mais avant tout la qualité et l'efficience du système d'éducation et de formation ". Cela vaut pour l'insertion des réfugiés comme pour les défis d'avenir pour la compétitivité à l'ère de l'industrie du futur. "Ce qui fait la croissance des économies modernes, c'est moins d'investir dans le béton que d'investir plus dans les têtes ».

En d'autres termes, conclut le Conseil des Sages : "L'économie allemande doit s'adapter à un monde de plus en plus complexe et en perpétuelle évolution ». C'est là une priorité absolue pour les responsables politiques car "il n'y aura aucune voie de retour possible permettant de préserver l'essentiel de nos acquis ». L'appel est on ne peut plus clair.

(I. B., 10/12/2015) 